\title{
The Organizational Valuation of Valuation Devices. Putting Lean whiteboard management to work in a hospital department
}

\author{
Amalie Martinus Hauge
}

\begin{abstract}
This paper is about the interplay between multiple modes of valuation. The paper engages with the question of how a valuation device intersects with the working values of an organization. While the many studies of valuation practices have drawn attention to the pervasive effects of valuation devices, only a few studies have taken into account the fact that many spaces, including organizations, are already filled with practices and ideas that constitute what is valuable. Revisiting classical organization theory, this paper shows that organizations comprise multiple, more-or less-integrated modes of valuation. Empirically, the paper draws on an ethnographic study of Lean management at a children's hospital, which is presented through analytical snapshots. The paper suggests that an organizational turn is relevant for valuation studies, as this first allows an analytical expansion to include less 'deviced' valuations, contributes to the ongoing culture vs. device debate offering an alternative to the causal analysis of devices and effects without making the 'ineffable culture' what makes or breaks the causality.
\end{abstract}

Key words: modes of valuation; organizational turn; organization theory; health care; Lean management; snapshots

Amalie Martinus Hauge, Department of Organization, Copenhagen Business School and Juliane Marie Centret, Rigshospitalet, aha.ioa@cbs.dk

(C) 2016 Authors

LiU Electronic Press, DOI 10.3384/VS. 2001-5992.1642125

http://valuationstudies.liu.se 


\section{Valuation Studies}

\section{Introduction: Bringing Devices to Work}

As part of a general tendency within the public sector, quantitative evaluations and performance measurements have proliferated in hospitals. Where quantification and numeric evaluation some years ago existed mainly in the context of the evidence-based medicine movement (Sjögren 2008, 368-383; Timmermans and Berg 2003), today, calculations and quantitative accountability are integrated in a number of hospital tasks. These include everything from quality work (Madsen 2015; Zuiderent-Jerak and Berg 2010) and patient involvement (Bech 2012) to management and coordination (Sjögren 2008). The increasing employment of tools of valuation has brought about new circumstances for the hospital: with the new measurements come new professional roles (Madsen 2015), new administrative tasks and new expenditures, to name a few. The increased use of quantitative tools of assessment has created an intensive focus on the importance of being able to measure the effects of efforts, but the interesting question is whether they have also played a part in changing what is regarded as important and valuable at the hospital.

The proliferation of tools of evaluation and quantitative assessments has sparked the emerging field of valuation studies (Kornberger and others 2015; Orlikowski and Scott 2013). Scholars engaged in a number of diverse fields (Lamont 2012) have shown how the introduction of valuation devices has had pervasive performative effects on the field, for example, how university rankings affect university management (Espeland and Sauder 2007), how fish quotas change a fish market (Holm and Nielsen 2007) or how shopping carts affect shopping choices (Cochoy 2008). By unfolding the sociotechnical arrangements of these devices, the studies show how the act of measuring, ranking or rating not only affects how the value of something is established but also affects what is considered valuableor what 'counts' (Dussauge et al. 2015). In this way the constitutive effects of valuation devices have been heavily accounted for, leaving the impression that the introduction of a new device will almost inevitably have dramatic consequences.

Few studies of valuation have provided alternatives to the almost causal narratives produced about the effects of valuation devices (Boltanski and Thévenot 2006; Fourcade 2011; Zuiderent-Jerak and van Egmond 2015). One of these is provided by Fourcade (2011). In her analysis of how we ascribe monetary value to an intangible thing such as nature, she argues that the answer to 'how' we do it is not enough; we should also ask 'why', which leads her to argue that national 'logics' or perhaps 'culture' encapsulates and frames how devices work (Fourcade 2011: 1770). Relatedly, Boltanski and Thévenot (2006) argue that people give worth to things based on a 'political metaphysics' of orders of worth. For both Fourcade (2011) and Boltanski and Thévenot (2006), the consequences of valuation 
devices therefore need to be found outside of those devices, in the 'cultural repertoires' in which such devices are situated. Although these authors present such a move as an important step for the study of valuations and justifications, other authors fear that this may lead to a rather traditional culture vs. materiality leapfrog discussions about what drives history (Zuiderent-Jerak and van Egmond 2015).

In this paper, I aim to contribute to studies of valuations and particularly the culture/device debate with an organizational perspective built on classical organization theory (see, for example, Simon 1964; Lawrence and Lorsch 1967; Barnard 1968 [1938]; Mintzberg 1979). Drawing on an ethnographic study of the use of Lean in healthcare, and more specifically the use of the Lean valuation tool 'whiteboard management' and how this is put to work in a department of neonatology of a children's hospital, I engage with the question of how a valuation device intersects with the working values of an organization. Through empirical analysis, I argue that valuation devices are rarely put to work in value-free realities waiting to be sorted and hierarchized. Taking inspiration from classical organization theory, I show that organizations are already filled with ideas about what is valuable, implicitly defined in the work of the organizational members. Depending on the tasks organizational members conduct, they assess the value of things with different 'grammars'. The paper suggests that an organizational turn in the study of valuations provides a valuable contribution to the culture/device debate, as it offers an alternative to the sometimes overly causal analysis of devices and effects, without making the 'ineffable culture' (Fourcade 2011: 1770; Zuiderent-Jerak and van Egmond 2015: 51) what makes or breaks the causality.

\section{Theoretical Framework: Modes of Valuation}

With the purpose of investigating how the valuation device of whiteboard management intersects with the working values of hospital organization, I employ the concept of modes of valuation. I use this composed concept similarly to Stark (2011) as a particular manner of assessing and attributing the value of something; but I develop it further by attaching four dimensions: A particular grammar of assessment, and a particular goal, task and time configuration, which will be presented in this section.

Valuation can productively be defined as 'any social practice where the value or values of something is established, assessed, negotiated, provoked, maintained, constructed and/or contested' (Doganova et al. 2014: 87) including (judging, improving, appreciating, and lots of other activities' (Heuts and Mol 2013: 141). While this is a rather broad definition, the dominant tendency is to study valuation performed in connection with a particular device in the sense of a 


\section{Valuation Studies}

specific socio-technical assemblage with a relatively unambiguous 'interface', such as a particular ranking (Espeland and Sauder 2007), set of guidelines (van Loon and Bal 2014), rating (Rona-Tas and Hiss 2011; Pénet 2015) or model (Cabantous and Dupont-Courtade 2015). Similarly, I began the ethnographic study of whiteboard management in the department of neonatology by investigating the question 'what does the whiteboard do here?' expecting to do a study on how the whiteboard's program of 'optimizing' value would have intended and unintended effects on the enactment of value in the department.

With time, however, it became clear that it was not only the valuation installed by the whiteboard that was interesting; the hospital's valuation of the whiteboard was equally relevant for investigation. Among other things, some nurses never attended because they could not leave their patients and some doctors again and again contested what the whiteboard manager suggested. To understand this, it was pertinent to develop an alternative to the dominant analytical strategy of studies of valuation that could grasp what happens as a valuation device intersects with the working values of an organization. The aim was to specify not only the device's means of working and its effects but also its relationship to prevailing tools and practices of valuation at play in the organization. For this purpose, I developed the concept of modes of valuation, drawing both on Stark (2011) and other lines of theory.

I conceptualize modes of valuations as consisting of four dimensions: Grammar of assessment, goal, task, and time, as depicted in Figure 1. Two of these are based on classical organization theory (goal and task) and two on a broader sociological/philosophical tradition (grammar of assessment and time). The idea is that these dimensions define a particular manner of assessing value-meaning that what is valuable is dependent on the mode enacted.

With 'grammar of assessment' I draw on the post-structuralist ideas of grammar in relation to subjectivity and discourse (Lévi-Strauss 1963; Foucault 1988; Lyotard 1993; Owen 1995: 489-506) and, similarly to Barley (1986: 83-84), use the concept to denote the principles and elements that constitute a particular 'frame' of valuation. In contrast to how Boltanski and Thévenot use the termgrammar (Boltanski and Thévenot 2006; Thévenot 2007, 2015), namely to describe a collectively shared framework of modes of justification on which persons can draw in situations of dispute (Boltanski and Thévenot 1999), I refer to the particular assessment system used by a specific mode of valuation.

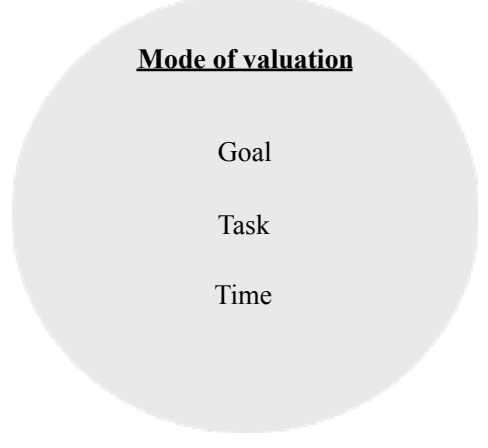

Figure 1. Grammar of assessment. Source: Author's illustration 
Concretely, this is constituted by the metrics, categories and reference points applied to assess the value of something and to prioritize between different choices. I use the notion of grammar rather than valuemeter (Latour and Lépinay 2009: 135; Zuiderent-Jerak et al. 2015: 119-135, esp. 135) or valorimeter (Latour and Callon 1997) to signify a system that is not exclusively based on a numeric measurement, as 'meter' suggests, but can also contain forms of assessment that are not based on numbers.

The notion of 'goal' is a classical element of organization theory (see, for example, Simon 1964; Schein 1965; Barnard 1968 [1938]) that generally refers to that toward which is aspired: For the whiteboard manager, this could be better results related to a particular Lean effort. The notion of 'task' is another classical element from organization theory generally referring to the actual piece of work that is conducted. In the hospital context, it can, for example, be to administer eye drops.

The 'time' dimension is inspired by some of the works by the Russian literary Mikhail Mikhajlovitj Bakhtin (Bakhtin 1937). Bakhtin uses the term 'chronotope' (literally time-space) to point to the interconnectedness of temporality and spatiality in literature. In this context, I use this idea (but only the time element of the concept) to connect temporality to modes of valuation with the purpose of showing that the way time works is contingent on what mode you are in. In this way, the time dimension is also similar to Lawrence and Lorsch's notion of 'time orientation', which they argue is defined by the 'definitive feedback of the relevant subenvironment' (Lawrence and Lorsch 1967: 8). Thus, a mode can, for example, be oriented toward the 'acute' in the immediate now or toward a more distant, plannable future.

I understand modes of valuation as dynamic and situated in concrete practices, constituting what counts as valuable. Modes of valuation are not necessarily defined by particular professions or persons; rather, actors can engage in different modes. For example, I see doctors as enacting one mode when they perform acute procedures and another when they discuss administrative issues. Modes of valuation are thus an approach to exploring how a valuation device intersects with the working values of an organization, as it allows for multiple and coexisting answers to how things come to count.

\section{Organization theory's relevance to studying multiple modes of valuation}

The conceptualization of modes of valuation is closely related to the idea that organizations are usually already 'filled spaces' (Vikkelsø 2010). A new valuation device will therefore typically have to compete with other valuations and find its place in the organizational order 


\section{Valuation Studies}

defined not only by devices with orderly interfaces but also by valuations that are not announced, not published and do not necessarily rest upon complicated numeric calculations but happen as part of the mundane conduct of the daily work in the organization. In organization studies, the integration and coordination between different subdivisions, professions or roles toward an organization's common goal is a classical theme. In valuation studies, however, the coexistence of and dynamics between different valuations is an aspect that so far has received only limited attention. In this paper I explore the value of drawing on organization theory to capture such dynamics.

Around the 1960s, organization scholars began to see organizations as open systems whose structure was contingent on the organizations' task environment(s) (Lawrence and Lorsch 1967; Scott 1981; Thompson 2007 [1967]). Complex task environments were reflected in differentiated organizations with various subsystems, which made coordination and integration a major managerial challenge and research focus. A good example of dealing with this is "Differentiation and Integration in Complex Organizations" (1967) by Lawrence and Lorsch. They understand integration as "the process of achieving unity of effort among the various subsystems in the accomplishment of the organization's task" (1967: 4) and argue that in differentiated organizations, integrative devices or personnel functions that work to integrate the different subsystems are necessary to achieving unity of effort. Such integrative devices must have six characteristics to be effective: (1) the device must have an intermediate position to the "subsystems" it aims to integrate; $(2)$ the influence of the integrator must derive from technical competences; (3) the integrators must perceive rewards as related to the organization's total performance; (4) the integrators must have high influence throughout the organization; (5) the influence must be on a requisite level; (6) there must be a developed means of conflict resolution (1967: 44-46). In the discussion, I will explore how this perspective is relevant to investigating the challenges that can emerge when multiple modes of valuation coexist.

\section{Methodology: Constructing Empirical Snapshots}

The ethnography on which this paper is based was conducted at the main hospital in Denmark. For this paper, I chose to focus specifically on the use of whiteboard management in the department of neonatology. This department is one of the most highly specialized departments of neonatology in Scandinavia, treating premature children; children with congenital malformations, heart diseases, neurological disorders, surgical illnesses; and children below the age of two with need for intensive care. This department was among the first in Denmark to work with Lean and whiteboard management. Their initiative to use Lean and whiteboard management was voluntary and 
has not been problem free, but it has generated educational experiences for themselves and other departments embarking on the use of Lean. I chose to focus on whiteboard management in the neonatology department because it constituted an elaborate exemplary answer to the question of how a valuation device intersects with the working values of an organization. Here, I was able to investigate how whiteboard management intersects with the values that are already at play and what organizational consequences this has.

For approximately one year, I explored the practice of whiteboard management at the hospital, and for three months, I focused specifically on the department of neonatology. In the empirical study, I relied on complementary sources of information. Most importantly, I observed the weekly meetings that took place every Tuesday at 9:05. Second, I shadowed doctors and nurses in the department and had walking talks with them about Lean and whiteboard management. Third, I conducted 11 one-hour-long qualitative interviews with central actors in the department, including the clinical managers (3), the Lean manager (1), head physicians with different roles (4), nurses of different ranks (2) and one secretary. Fourth, I gathered documents related to the department's Lean work and took photos during my observations, which I used as reference points during the interviews.

I have presented the findings of the study as analytical "snapshots" (Running 1997): Detailed descriptions with the purpose of conveying a distilled flavor of what is going on in the department. The snapshots are not identical to my observations, yet they allow for a more accurate description of the atmosphere and the tensions in the department than would a few citations or long, less edited field note extracts. Additionally, the snapshot format matches my interests in exploring modes of valuations, as it makes it possible to juxtapose different examples of how things are made valuable.

\section{Analysis: Introducing the Valuation Device Whiteboard Management}

In 2011, the clinical management board decided to apply Lean to free more resources for research and other core activities. Among other things, the department employed a Lean manager (a nurse with training as a Lean consultant) who, in collaboration with the quality coordinators and the clinical management team, has conducted weekly whiteboard meetings ever since. However, what was supposed to be a common strategy of enhancing value and creating flow almost immediately became a highly contested activity in the department of neonatology. In the following, I unfold the activity of doing whiteboard management. Whiteboard management is a carefully orchestrated activity, where the elements on the whiteboard as well as the articulation work by the meeting conductor are meticulously 


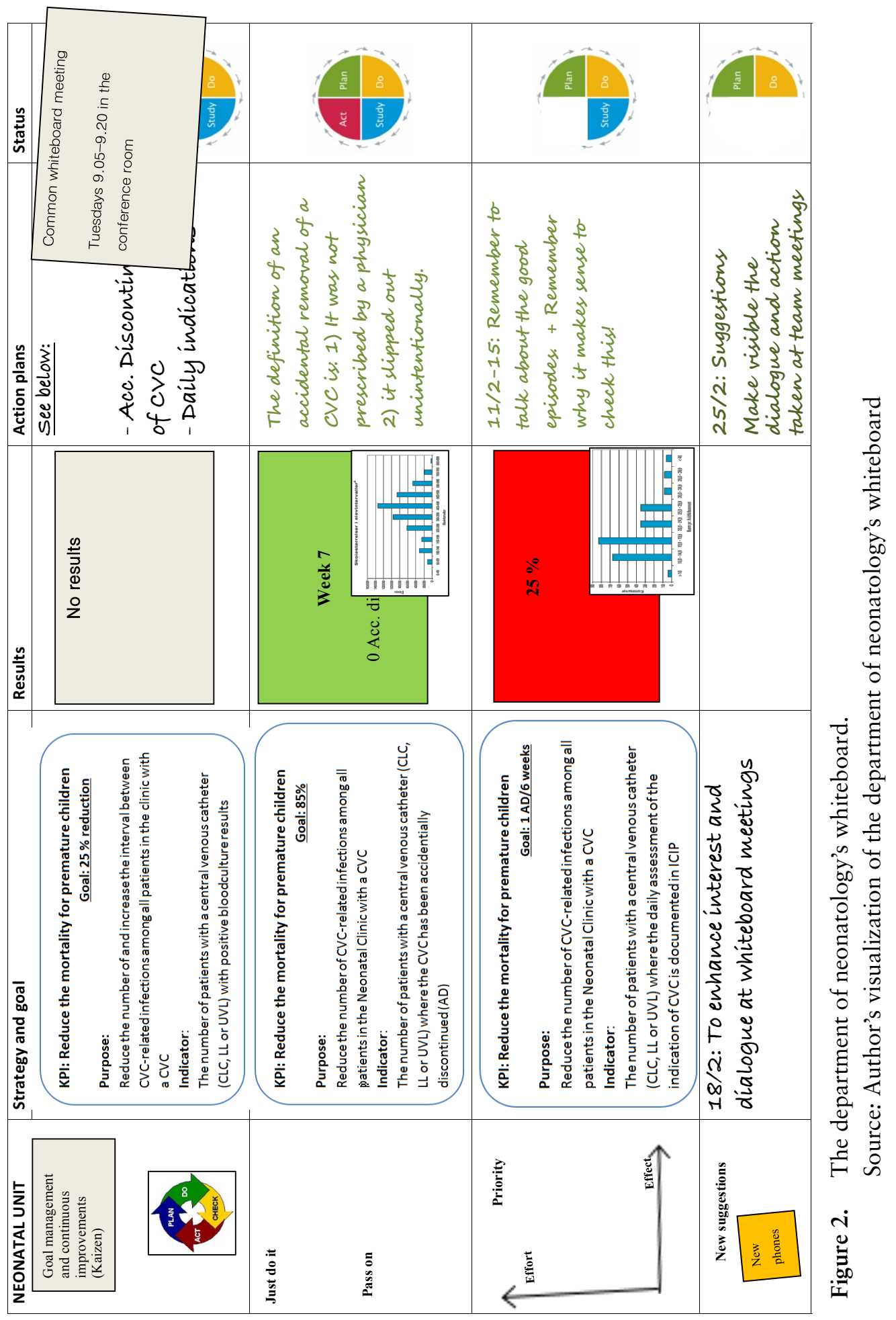


choreographed. In a neighboring department, I witnessed how a Lean consultant coached a future whiteboard meeting conductor on how to perform whiteboard management:

The whiteboard must be alive! Update the numbers every week; no one is interested in old numbers. It attracts attention when you focus on the core tasks. As an example this can be re-operations. They attract people! [...] And never put up a red result [on the whiteboard] without relating an "action" to it. Also: There has to be a whiteboard conductor. One who leads the meeting. Another should be appointed writer. You cannot write when you lead! The amount of people should make it possible for everybody to hear. If there are too many people, then you should conduct meetings in different fora.

The citation shows that whiteboard management relies on a combination of two central mechanisms that respectively aim to (1) bring the organization to the whiteboard; (2) bring the whiteboard to the organization. The first mechanism involves the creation of a specified space in front of the whiteboard where people stand close together at a certain time with a certain frequency. It is not that the department does not have meetings already: Among others, there are morning conferences in the different teams (there are three specialized teams in the department), among the different professional groups (nurses, physicians and secretaries) and among different crossfunctional groups (the quality board, etc.). However, the whiteboard meetings in the hallway are different in the sense that they seek to gather department staff across the different sections to coordinate activities toward shared goals.

The second mechanism involves the translation of the common goals into particular tasks, which are systematically monitored against key performance indicators (KPI) and adjusted if the objective is not achieved. This mechanism is about linking the activities at the whiteboard meetings to the work done in the department, which is what I call "bringing the whiteboard to the organization." In the following, I will present the details of the whiteboard.

The department of neonatology's whiteboard (Figure 2,) sits between the entrance to one of the hallways and the elevator housing. The basis of the whiteboard is columns and rows marked by colored adhesive tape, attached plastic folders and headlines written with permanent marker. From left to right the board reads as follows: The first column is for suggestions. These can be formulated by members of the department on post-its and placed on the bottom of the column. In conversation facilitated by the Lean manager, the suggestions are prioritized using the depicted coordinates system, which shows that suggestions that require "low effort" but deliver "big effects" are best.

The next column contains the objectives on which the department is currently focusing. On this whiteboard projection, three objectives are on the board, which all relate to the KPIs "to reduce the mortality rate 


\section{Valuation Studies}

for premature children." In addition to these three, a fourth handwritten objective is to "enhance interest and dialogue at meetings." According to the Lean consultants, all staff in the department should be able to relate to and affect the objectives on the board, even though their specific tasks differ. To find an objective that fits this format is no easy task: "Even at Novo Nordisk they spend as long as three months developing the right objective," a Lean consultant says to calm down another clinical director, who is frustrated that her department's whiteboard meetings do not go as planned. This difficulty is also reflected in the department of neonatology, as we see in this extract from a workshop on the department's strategy day:

Department Director (DD): We need to make our KPI more concrete.

Head Physician 1 (HD 1): We only measure infections in relation to the KPI.

HD 2: We have to have a higher time resolution! We need to translate the work flows of the big out-patient departments to our context. Think in processes.

DD: We should use the meetings to create a flow [...] not just the data, but also the meetings. That makes sense.

Head nurse $(\mathrm{HN})$ : Yes, but we also have to find it important. It should be meaningful!

Section nurse: Yes. But what can it be?

HD 1: Take mortality-what we can monitor easily?

HD 4: We could take weight fluctuations as an indicator of the fluid balance?

Senior HD: It is important that we don't guess. We have to do it evidence-based.

HD 4: Sure sure-it was just a suggestion—something like that!

HD 2: What about something with blood sugar fluctuations?

DD: We need to remember the multidisciplinarity-the objective must be something that we can all relate to (transcription extract).

In this case, the department's quality group continues the complicated process of developing an objective that lives up to the criteria of having the right time resolution (HD 2), being important and meaningful (HN), being evidence-based (senior $\mathrm{HD}$ ) as well as relatable for all department members (DD).

The third column on the whiteboard is for results. As the Lean consultant states above, the results must be updated every week before the meetings in order for the participants to be able to see if their 
actions of the current week have made a difference. This demand increases the complexity of deciding on an objective, as the data about the objective have to be compatible with the whiteboard meetings' weekly frequency. If an objective is achieved, the result is printed on green paper. If not, the paper is red. The fourth column is for "actions." Participants have to come up with actions if a goal is not achieved. When conducting the meeting, the whiteboard manager goes through the results, and in announcing a red result, she will ask the participants in the meeting to come up with suggestions on how to achieve a better result next week. For example, as part of the discussion related to the red result (row three) about how to improve registrations of catheter assessments, a head physician suggested "sharing stories in which it made a difference to make the registration" (suggestion noted in row three, column four). The last column indicates the status of the goal using the PDSA-circle (is the effort Planned, Done, Studied and Acted upon?). The principal goal of the whiteboard is thus to work toward the KPIs by engaging participants in turning the red results green.

\section{Bringing the Organization to the Board}

The logic of whiteboard management is that as long as the objectives on the board are important to members of the department, they will participate in the meetings. However, this was not the case in the department of neonatology. In fact, participation became a source of great frustration in the department, for some because they wanted more participants, for others because they did not see the point of participating and for others because they could not participate and felt left out. In spite of the meetings' mandatory status, the amount of participants at the meetings varied extensively, and not infrequently the meetings were cancelled. In this section, I look into the mechanism of bringing the organization to the whiteboard by analyzing how varying importance is attributed to the whiteboard meetings depending on the mode of valuation enacted.

Mode 1: The Lean manager gathers participants

It is 9 a.m. The Lean manager enters the hallways of the department of neonatology to remind people that the weekly whiteboard meeting is beginning in five minutes. The atmosphere in the hallways is characterized by brightly lit effectivity, emphasized by the dim quiet oozing from under the doors of the patient rooms. A phone is ringing; a nurse is fetching medicine with fast and experienced movements; two young physicians are looking at some scans speaking in low voices; a newborn is transported to the operating theatre under piles of machinery, and the first visitors are searching for the button that opens the automatic doors. Two head physicians concentrated on their conversation pass by with their eyes fixed on the papers in one physician's hand. The Lean manager continues down the hallway and looks into the small windows or the 


\section{Valuation Studies}

half-open doors of each patient room to assess whether the situation allows for her to enter and inform staff about the forthcoming meeting. In the hallway she makes eye contact with a nurse head of section and taps her wrist watch. "Oh yes. Two minutes," says the nurse head of section and continues in the other direction. Returning to the whiteboard from her round in the department, the Lean manager goes through the numbers one last time with the nurse responsible for quality to make sure that everything is updated and ready for the meeting. They hope that many physicians and the clinical director will participate, as the most burning issue requires the physicians to take action. At 9:05 she looks at the clock above the door, tightens her lips and bids the first arrivers welcome.

\section{Mode 2: A head physician and a nurse are attending to critical patients}

The morning conference is over and head physician Jane is walking down the hall to the changing room. It will be a busy day, according to the clinical director, who ended the conference by proclaiming that they must discharge as many patients as possible to make room for those that will arrive. A nurse stops Jane in the hallway and wants a consultation about a patient. Jane says she will come back as soon as she is in her uniform. The nurse goes back into the room behind the reception preparing for the consultation by reading the patient's records over again. She waits for longer than expected. A colleague comes running frantically looking for something. "Have you seen Omar's file?! It is urgent!" After a frantic search, they find the file and the colleague calls Omar's parents, who have slept at home tonight. After the conversation she hangs up and says "that is the worst of messages; to tell parents that they need to come as fast as they can.” After a while, head physician Jane comes to get the nurse: "Omar's heart stopped so I had to run in there right away-now, let's talk." After the consultation, head physician Jane continues toward her next patient, Albert. On her way, she meets the specialized nephrologist, and while walking, they discuss what to do about Albert's, sudden, critical fluid retention. In the hallway, she passes the Lean manager. However, by the pace of her walk, her waving coat and the firm grip around the file in her hand, everybody knows that head physician Jane has more urgent things to see to.

\section{Mode 3: A nurse is caring for baby Emily}

In the patient rooms, the light is dim and movements are slow paced. Sleepdeprived parents are rising from their beds, and some are having breakfast. Nurse Mary converses with the parents in familiar terms about how the night went and admires a mother's knitting project. The baby cribs with home-made nametags and teddy bears testify to the length of some families' stay in the department. Mechanic, monotone sounds from a ventilator and a dialysis machine mixed with the frequent offset of alarms are a constant reminder of the gravity of the families' situations. Seemingly asleep, baby Emily lies on her back in a white crib with adjustable height and wheels for transportation. Nurse Mary leaves the room to fetch the eye drops. On her way to the room-sized medicine cabinet, she meets a colleague and asks how "her little friend" is doing. All the nurses refer to their primary patient as their little friend. "Stable, but waiting for a new brain scan," the colleague says. As Mary returns to Emily's room, she passes the Lean manager, and they quickly catch each other's eyes. In the patient room, Emily's father looks up from his phone to see who entered the room, then looks down again. For the fourth time this morning, Mary applies the hand sanitizer from the container next to the door, and asks if either of the parents would like to put the 
drops into Emily's eyes. The mother shakes her head. Mary doesn't go to the whiteboard meeting that day.

\section{Mode 4: A head physician is preparing for the whiteboard meeting}

Head physician Dan met earlier than he had to today, because he wanted to get some paperwork done. In addition to the ordinary work, Dan is the manager of the "transportation team," which is available 24/7 if other hospitals in Denmark need to have a child transferred to Hospital $\mathrm{H}$. He is also part of the team responsible for the medical apparatus and utensils. The department has recently changed suppliers for the bandages used to hold catheters in place. Today, Dan is interested in how his colleagues-especially the nurses-have experienced the new bandages, which are cheaper and, according to a new study, just as good as the old ones. He hopes they will share some experiences at the whiteboard meeting. He is also interested in the follow-up to the discussion about whether or not the attending physician on night shift should take on a new area of responsibility: To create an overview of the patient situation and hand it over to the day shift. $\mathrm{He}$ thinks it is unreasonable to put more duties on the night shift, and he has planned to stand up against this idea, even if the Lean manager and head nurse are still behind it. He prints out some information about the new bandages before he gets up and taps his front pocket to see if his notepad and pen are in place. $\mathrm{He}$ reminds his younger colleague using the computer next to him about the meeting. Having walked the short distance to the whiteboard, he looks at the updated numbers one by one. The clinical director arrives and the head physician asks what the director thinks about the idea of adding more areas of responsibility to the already burdened night shift attendants. Then the Lean manager says "welcome" and they quietly finish off their conversation to focus on the Lean manager from their position in the back of the crowd.

\section{Juxtaposing Snapshots: Multiple Versions of the Value of Participating in Whiteboard Management}

The four snapshots (Figures 3-6) portray particular modes of valuation. It is clear that the value of participating in the whiteboard meetings is assessed in different ways and with different results. Depending on the task, time and goal orientation of each mode, a certain "grammar" is enacted through which the value of participating in whiteboard meetings is assessed: In the first snapshot, the Lean manager sets out to remind nurses and physicians to participate in the meeting. In the practice of whiteboard management, participation in whiteboard meetings is conceived of as very valuable: Whiteboard management is the means of working toward the KPIs of the department. In the snapshot, we see how the whiteboard manager performs an ongoing assessment of the possibility of almost every colleague she meets participating in the meeting. While the

Mode: whiteboard mgt

Goal: Improve KPI results

Task: Conduct whiteboard meetings

Time: Weekly cycles

Figure 3. Grammar of whiteboard management. Source: Author's own work. 


\section{Valuation Studies}

Lean manager enacts a mode where it is highly valuable to get participants to the Lean meetings, she is not submerged in the mode to the extent that she does not also recognize that there are other modes at play in the department in which participation is not assessed as valuable or possible.

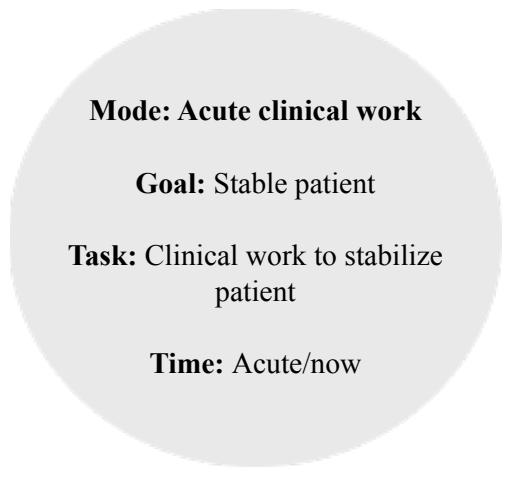

Figure 4. Grammar of acute clinical work.
In the second snapshot, the head physician is too busy with acute clinical work to attend the whiteboard meeting. For her, in Room 5 it is valuable to find a solution to Albert's critical fluid retention and to keep Omar in Room 2 stable until his parents arrive. There is thus clearly misaligned time orientation. This is clear to the Lean manager, who does not try to remind her of the meeting.

Source: Author's own work.

The third snapshot portrays how participation in whiteboard meetings is prioritized in the practice of nursing. Here it becomes almost impossible to participate -and also almost unnecessary. In the mode of nursing, nurses often have to be physically present in patients' rooms all the time. If you go to the bathroom, you ask a colleague to attend to your patient. When caring for less intensive patients, nurses are able to participate in the meetings, but it is not necessarily seen as more important than talking to families or giving eye drops.

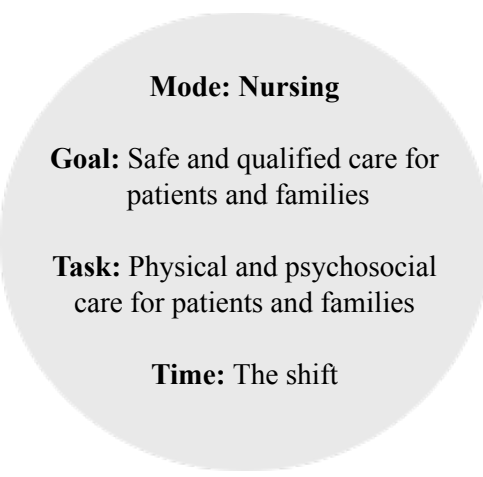

Figure 5. Grammar of nursing Source: Author's own work. Accordingly, attending the whiteboard meetings is not purposely avoided, but neither is it conceived of as necessary to conducting the main task of caring for the patient.

Mode: Admin. work

Goal: Best-practice, evidence-based and standardized departmental practice

Task: Organize and facilitate departmental work

Time: Future

Figure 6. Grammar of

administrative work. Source:

Author's own work.
In the fourth snapshot, we follow a physician preparing for the whiteboard meeting. Similar to the head physician attending to Omar's cardiac arrest, he takes care of many acute problematics during the day. However, due to his extra responsibilities, he is also enrolled in tasks that require an overview and a more longitudinal perspective of the depart- 
ment, for example, to know if remedies are used properly or if orders or regulations need modification. In the mode of solving such tasks, time orientations are more aligned and Lean is regarded a useful tool, although with the remark that Lean should not be used "too insistently." In the words of another physician with organizational responsibilities, it is important to use Lean with a sense of humor; otherwise, people will be fed up with hearing about the wonders of Lean. In this mode of planning and coordinating, he believes that Lean and the whiteboard meetings are useful tools (only) to the extent that they do not exclude, disunite or upset people in the department. If so, they are at risk of dismantling the planning and coordinating qualities of Lean whiteboard management, which are the qualities he finds valuable.

In order for whiteboard management to work as intended, the department members enacting the modes of valuation presented here are among those who must be "brought to the board" for whiteboard management to work. This section has given some insights into why this is a challenge in the department. However, to bring people to the board is not enough for the whiteboard to work: actions decided upon during the whiteboard meetings must also be carried out in the department.

\section{Bringing the Whiteboard to the Organization}

To investigate the challenges of bringing the decisions from the whiteboard meeting to the department, I focus in on a concrete objective on the whiteboard, namely the objective of improving adherence to the rule of daily assessment of the relevance of the patients' central venous catheters (CVCs). This is depicted in Figure 7, which zooms in on this particular goal on the whiteboard. For the sake of simplicity, I adopt the department's own nickname for this objective: "The indication".

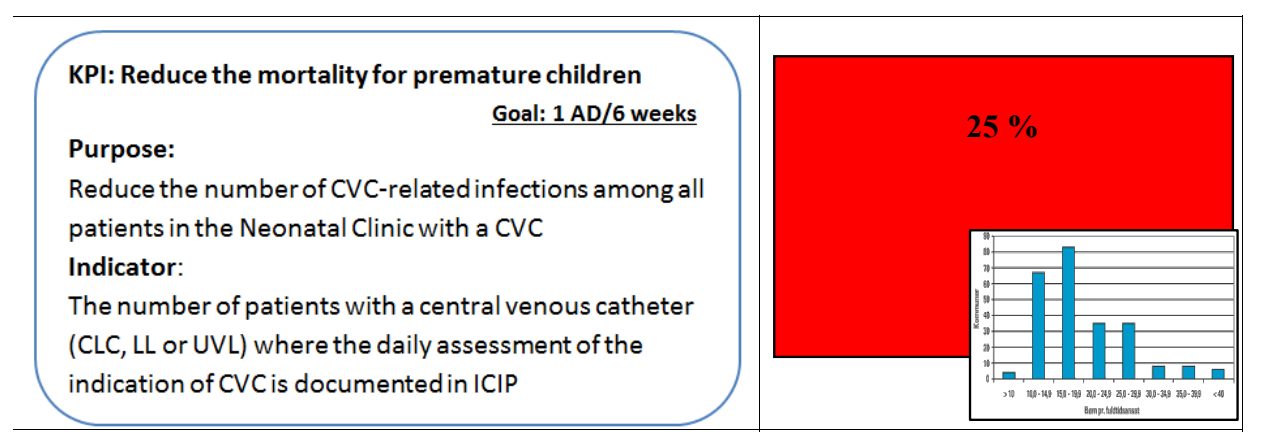

Figure 7. A whiteboard objective. Source: A zoomed in image of Figure 2

Let me provide further detail: CVCs are inserted into the veins of a patient to administer medications and fluids, obtain blood tests and/or 


\section{Valuation Studies}

measure central venous pressure. While in most cases CVCs are of utmost importance, CVCs also entail an increased risk of the patient getting an infection. This risk increases with the period during which the CVC is inserted. Thus, the purpose of daily assessing the continued relevance of the CVC is to make sure that only those patients who need a CVC have one and that the CVCs are always removed as soon as possible. The means of measuring whether the assessment is taking place is to ask doctors to tick a box in the electronic hospital record ICIP (IntelliVue Clinical Information Portfolio) when they have performed the assessment. The department quality board counts and compares the number of ticks to the overall number of patients with CVCs. The percentage of assessed CVCs is printed on a sheet of paper and placed on the whiteboard. While department members support the KPI of reducing the mortality rate, the task of making the indication creates tension in the department week after week.

The following is an example of the challenges related to the implementation of the indication. At a Lean whiteboard meeting in February 2014, the result of the week's indications is yet again printed on a red sheet of paper, showing the same result as the previous weeks; that the objective is not achieved. The head physician, who is also part of the quality board, reads aloud what the whiteboard already states: Physicians have indicated that they have assessed the relevance of the CVC in only approximately 25 percent of cases. This is significantly below the declared goal of 85 percent. The atmosphere in the meeting is tense, and it is clear that the indication is not regarded as equally important by all members of the department. Different valuations of the indication are reflected in the following statements:

1. Head physician with administrative responsibilities: I think the problem stems from the fact that the box to be ticked is located in a place in ICIP where no one operates. However, now that the fluid ordinations are also going to be there, all of a sudden it will be a process that makes sense.

2. Clinical director: I think we have to reconsider one more time. We have to remember that it is not the tick itself that improves the child's situation. What is important is that we don't have catheters inserted longer than necessary. So we must reflect: Is this worth spending so many resources and so much time on this registration? Is this how we secure the best treatment for patients?

3. Head physician, quality board: The indication of the CVCs is a thing that simply MUST be registered. Not that I am too good at it myself, but it MUST be done. It is one of the few things we have evidence to support. The longer they are inserted, the greater the risk of catheter infections. They need to be removed as soon as they can.

4. Head Nurse: There is anarchy here: Why keep spreading doubt-"is it really necessary and is it a good parameter?"-Why not say: This IS the way [of achieving a reduced mortality rate]. We HAVE to do it. It is what we have decided 
in the clinical council and on the quality board, and now we SIMPLY have to do it.

5. Nurse from section with typically non-intensive patients: On the other hand I think that when we do rounds, it has become something we remember and talk a little about like "So the child gets this and that [through the catheter ]" So we do talk more about it than if we never focused on it.

The statements show the dissonant assessments of the value of making the indication. In the first statement by the head physician, the lack of indications in ICIP is categorized as a practical problem: His anticipation is that when doctors get an additional task in ICIP, they will make the indication. In the mode of administrative work, he regards the indications as a valuable means of getting an overview and of reaching the objective of 85 percent. In the second statement, the clinical director shifts the premises of the question and asks if spending time on making indications is how we secure the best treatment for patients. In this question he mobilizes the mode of acute clinical work: With the limited time we have, which is more important, treating children or making-and discussing-computer clicks? Assessed with this grammar, the value of making indications is doubtful. In the third statement, a head physician who is also on the quality board, argues in contrast that it is not good enough to omit the indication and only make the assessment. He assesses the relevance of the tick on the basis of international literature on how to decrease the mortality rate. On this basis, the indication as a means of making sure that the CVCs are removed as soon as they can be is one of the few things we have evidence to support and thus very valuable. This doctor is thus much more aligned with the mode of whiteboard management both in terms of goal and time orientation: CLC assessments must be indicated every day.

The fourth statement by the head nurse is also in support of making the indication. In alignment with the mode of whiteboard management, she argues that many arguments and reflections have preceded the decision of making the indication a whiteboard objective, and now it is a matter of doing as has been decided: Make the tick in ICIP. Spreading doubt about the relevance of the indication is time poorly spent and undermining the department's ability to reach its goal. In her statement is also a trace of an historical dispute in the department, where nurses have tried to implement new initiatives and doctors have declined to adhere to them. In working to solve the task of improving the organization and quality of the department's work, she firmly insists on bringing the whiteboard to the organization; doctors should make the indication. It is a decision they have participated in making, it is evidence-based and it is a means of reaching the goal of decreasing the mortality rate. The fifth and last 


\section{Valuation Studies}

statement by the nurse claiming that they do in fact talk more about it shows that making the assessment can be aligned with the time orientation of nursing: It can be a part of "doing rounds" which the doctors and nurses do together every day. On the other hand, it is something that they "talk little about," not something of utmost value when nursing. Additionally, nurses are not authorized to make the indication themselves, and for nurses, taking care of intensive patients and therefore not participating in whiteboard meetings, the focus on the CVC assessments is not necessarily known. While not regarded as a hindrance in the mode of nursing, neither is the indication of particular value in accomplishing tasks related to nursing.

While it has been decided to put the objective of improving the number of indications on the whiteboard, it is clearly a challenge to bring decisions from the whiteboard to the department. None of the modes of valuation reflected in the statements presented here is misaligned with the goal of making the indications: To reduce the mortality rate. However, as translated by the whiteboard, this goal becomes achievable only through conducting a certain task-to make the tick. To make the tick, department members have to change the way they normally do rounds by extending the time and attention given to the CVCs and, more problematically, the time spent on the computer. Consequently, the indication loses value in comparison to what department members could otherwise spend time on. As a head physician tells me in an interview: "It is as though focus on the whiteboard makes important aspects of the job secondary. The whiteboard meetings are the only mandatory meetings in the department, and all we talk about is quality and cleaning and stuff like that. We don't talk about patients and treatment. Not at all!"

In this case the practice of whiteboard management molds the department's goal and links it to a certain task and to a certain temporal configuration, which makes it misaligned with some modes of valuation in the department. In this way, whiteboard management both adds to the organizational misalignments of the department and magnifies its historical conflicts. Thus, whiteboard management fails to commensurate the modes of valuation in the department toward a united goal, but rather comes to increase the complexity of its coordination problems.

\section{Discussion: Organizing Multiple Modes of Valuation}

Having engaged with the case of Lean whiteboard management at the department of neonatology, I have illustrated how it may look when a valuation device intersects with the working values of an organization. On a more general level, this analysis allowed me to explore why it is useful to study valuation practices or devices in the "filled spaces" that organizations constitute. To further elaborate on the relevance of an 
organizational perspective to valuation studies, I will show how Lawrence and Lorsch's (1967) study of the role of integrative devices in achieving unity in an organization has something to offer in investigating the dynamics between coexisting valuations. I will do this by discussing whiteboard management as coming to act as an integrative device and highlight some of the challenges it faces in this endeavor. Lawrence and Lorsch argue that integrative devices must have an intermediate position to the subsystems they seek to integrate. Their study showed that organizations experience the most difficulty in obtaining an intermediate positioning in terms of (1) time orientation and (2) goal orientation. In what follows, I will briefly discuss, first, the challenges related to the time orientations of the whiteboard in comparison with other modes of valuation, and second, challenges related to the goal orientation of whiteboard management in comparison with other of the department of neonatology's analyzed modes.

By exploring the organizational valuations of whiteboard meetings with the theoretical framework of modes of valuation, we sawamong other things-how the time orientations of the different modes played a substantial role in shaping the grammar of what was valuable in the particular mode. This is summarized in Figure 8.

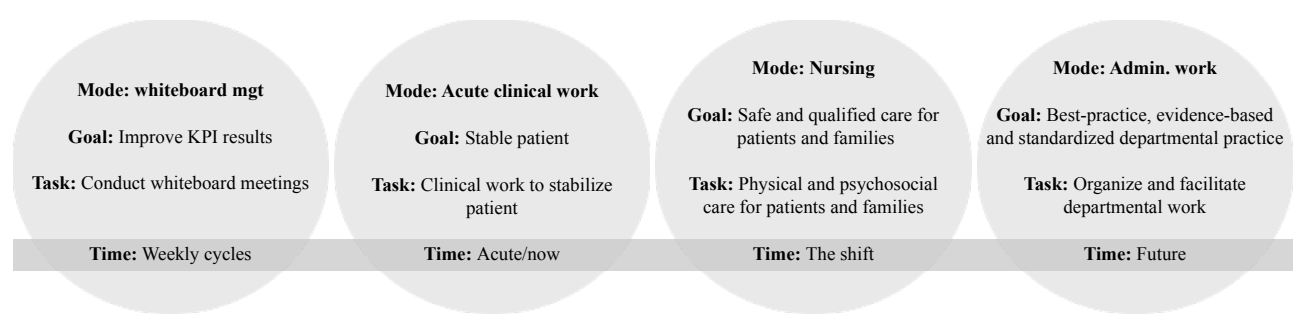

Figure 8. Comparing modes of valuation. Source: Author's own work.

Whiteboard management as a mode of valuation has a weekly time orientation. Feedback is provided every Tuesday when the results are printed on red or green paper. In the mode of acute clinical work, the primary feedback is instant-although clinical work done in acute situations may show results later on as well. In the mode of nursing, the main time orientation is the shift: The nurses conduct the plan of the shift as decided on in the morning conference (in the case of the day shifts), and they care for the patient to whom they are assigned for the duration of the shift. In the mode of administrative work, the time orientation is mainly toward the future: How can the department improve its statistics this year compared to last year; what can we do to solve the problem that too few nurses have intensive care training, etc.? Feedback for these types of issue varies but is generally more future-bound than other practices in the department. This time 


\section{Valuation Studies}

orientation of whiteboard management differs from the time orientation of, in particular, acute clinical work, but also from that of nursing. When the objectives and actions defined at the whiteboard meetings are temporally configured in a way too different from these modes, the argument based on Lawrence and Lorsch's article would be that it complicates integration because organizational members may not find feedback in other temporal structures equally valuable.

In terms of goal orientation, the goal of whiteboard management is to improve the department's KPI results: To decrease the mortality rate for premature children. This goal is not so far from the goals of "stable patient," "safe and qualified care" or "best practice" associated with the other modes of valuation in the department. However, as this goal is translated into the task of making ticks in ICIP to indicate the continued relevance of the patients' CVCs, it seems to create significant challenges in terms of integrating the different modes toward this goal. When this task is assessed through the grammar of, for example, acute clinical work, it is not necessarily aligned with the goal of decreasing the mortality rate of premature children. Physicians or nurses enacting the mode of acute clinical work may not always find it meaningful to spend time on opening ICIP and making indications. This underscores a central observation: While the goal of whiteboard management is relatively aligned with the goals in the other modes of valuation in the department, its link between goal and task is less intermediary to the other modes. In fact, whiteboard management is the only mode of valuation in which the practice is a means of achieving the goal. This explains some of the difficulties of bringing the organization to the whiteboard.

In spite of these challenges, whiteboard management manages to optimize certain things. When department members from different teams and professions get together and talk about their work, a number of ambiguities and tensions between different ways of doing things emerge. For example, at one meeting it turned out that two teams measured the placement of a CVC in different ways. This made it difficult to assess whether the CVC was placed correctly, because it was impossible to know if a difference in the measured position of the CVC was as a consequence of different measurement practices or an indication that the CVC was slipping out and needed replacement or adjustment.

While trying to work as an integrative device-or phrased more in the spirit of Lean-offering itself as a means of streamlining the department's work toward the common goal, whiteboard management rather comes to enact an additional mode of valuation. Whiteboard management becomes its own practice with a particular time orientation and a particular link between goal and task, and as such comes to add to the number of modes that need to be integrated in order to make the department work as a coordinated entity. Rather 
than making the department "lean" in the mundane sense of the word, whiteboard management comes to increase the organizational complexity of the department. The effects of working with Lean whiteboard management in the department are not that the entire department's view of what is valuable changes. The effects may unexpectedly turn out to be increased complexity rather than streamlined unity.

\section{Conclusion and Implications: When Valuations Intersect}

When a valuation device is put to work in an organization, its effects are likely to deviate from how it was designed. In the organizational arrangement the device intersects with other modes of valuation, enacting different ideas about what is valuable or important. Thus, the course of a valuation device is not defined only by the design of the device but also by how it is itself valuated by the prevailing or coexisting modes of valuation. When a mode of valuation (a grammar of assessment, a task, goal, and/or time orientation) enacted by a device deviates from existing modes, a consequence can be that the device is ignored or distorted. It can also result in new and unintended effects co-created in the intersection between the modes of valuation, as the example of diverging measurement practices shows. To expand the understanding of valuation devices regarding their impact, this paper has shown that an organizational turn in valuation studies is a productive way forward. Such a turn has (at least) two related implications for further studies of valuation.

First, an organizational turn would constitute an alternative to the dominant analytical approach of investigating the performative effects of a particular device without looking at the "scene" in which the device is put to work. To be able to analyze the scene, I have shown how strategies and learning points from organization theory are highly useful, as they offer a focus and vocabulary with which to engage and explore this scene. Furthermore, it implies a more symmetrical approach about which valuations to study. Currently, studies of valuation tend to prefer to study the most highly "deviced" modes of valuation. While these often provide neat interfaces and numeric figures that perhaps make them more appealing to address in analysis, it does not mean that they are necessarily more influential or relevant on the empirical scene than others. In this paper, this is illustrated by the fact that even when Lean whiteboard management comes with numbers, managers, meetings, resources and political backing, it does not make the doctors make the indication or show up to a meeting if they find other things more valuable.

Second, an organizational turn would be a way of pushing forward the culture vs. device leapfrog debate of valuation studies (Zuiderent- 


\section{Valuation Studies}

Jerak and van Egmond 2015: 45-73). The current debate is split between those who give primacy to culture or almost metaphysical orders (Boltanski and Thévenot 2006; Fourcade 2011: 1721-1777) and those who give primacy to particular devices (Callon 2007: 311357). An organizational turn as presented in this paper provides the possibility of taking in that with which the device interacts in a way that is practice-oriented and material. Rather than conceptualizing what is outside of devices as Culture with a capital C, this paper suggests that-in organizational arrangements-"it" may be approached as practices enacting valuations: Conduction of a task, striving after a goal and a particular time orientation amounts to a particular grammar through which the value of something is assessed. In this way, studies of valuation would gain a supplement to the approaches that (over)emphasize the agency of devices (ZuiderentJerak and van Egmond 2015: 45-73, esp. 50) while maintaining an interest in concrete, empirical practices where "the value or values of something are established, assessed, negotiated, provoked, maintained, constructed and/or contested" (Doganova et al. 2014: 87-96, esp. 87).

While this paper argues that an organizational turn is highly relevant for valuation studies, this does not imply the view that organization theory should uncritically be "added" to valuation studies. The combination also points to some of the areas to which organization theory has devoted less attention. This includes the role of devices-or technology-in relation to organizational goals. When technology is addressed in organization studies, with few exceptions (see, for example, Orlikowski and Scott 2013), this is mostly by interpretative approaches such as sense making or institutional theory, which typically do not address the "agential" qualities of technology. The contingency school (Lawrence and Lorsch 1967: 1-47; Thompson 2001 [1967]) perhaps most directly addressed technology's influence on organizational goals. They emphasized the way in which exogenous or "environmental fluctuations" (Thompson 2001 [1967]: 278) could "distort" the rational goal-achievement of technologies. Yet, even if it operated with a "bounded rationality" (Simon 1957; Thompson 2015 [1967]), meaning that the organization cannot comprehend all "possible sources of variation" (ibid.: 364), the contingency school did not address the agential role of technology in creating these "distortions" or in even establishing the goals.

Organization theory in general thus has something to learn from the way valuation studies address valuation devices. Devices in valuation studies are much less solid and more "mutable" and "mutating" than in organization theory. Rather than asking how well a means (technology) serves to achieve a goal, valuation studies would ask how a goal is established and what role technology plays in this establishment, not assuming that the goal preceded or is separable from the technology. One of the sources of inspiration for valuation 
studies, Latour, and his co-author Venn (2002) argue that "technologies never truly appear in the form of means" (2002: 248). Using the example of a hammer, Latour and Venn show how time and space is folded (referring to Deleuze 1993) into the hammer, making it an "end" of an historical and material development process at the same time as a "means" of a handyman. This makes it impossible to say that the hammer merely "fulfills a function." Rather, Latour and Venn say, the hammer alters the man who holds it in his hand making him envisage a "flux of new possibilities." An example of this from this paper is when it becomes a whiteboard goal to "enhance interest and dialogue at the whiteboard meeting." What was before a means (whiteboard meeting) of reaching a goal (decreased mortality rate), becomes a goal in itself due to the alterations installed in relation to the technology. From this way of approaching the role of technologies in many studies of valuation practices, organization theory could learn how elements of grammars of assessment, such as specific practices of goal, task and time, may become enacted differently and enter this flux of new possibilities.

For research on Lean management, this conclusion provides an alternative to the typical conclusions of implementation studies. Often, scholars describe the implementation of Lean in hospitals as failing due to "barriers" or lack of dedication (Brandao de Souza 2009: 121; Edwards and Nielsen 2011; Radnor 2011: 89-90; Radnor et al. 2012: 364-371). These studies' conclusions rest on the idea that Lean as a policy or design holds the correct or most valuable solution, and that practice distorts or ruins this solution. In this paper, I have aimed at shedding new light by approaching the hospital department as a filled space where multiple modes of valuation are at play, each with sensible ideas about what is valuable. In showing how Lean whiteboard management competes with other modes of valuation about how to organize the department's work, it becomes clear how the course of a device (or a policy) is shaped by its intersection with prevailing modes of valuation. This implies the relevance for hospital managers or Lean consultants of developing valuation devices close to practice, as this is where the organizational valuation of new devices takes place. Additionally, the organizational perspective to valuation implies that implementation of Lean is not mainly approached as a question of overcoming barriers to optimize value, but rather of how to organize Lean in relative alignment with what is constituted as valuable in the existing modes of valuation of the hospital. 


\section{Valuation Studies}

Acknowledgements. The author would like to thank the editors of Valuation Studies, two anonymous reviewers, Signe Vikkelsø, Center for Health Management at CBS, Bent Ottesen and Malene Mols from the Juliane Marie Center, the organizers and participants of the "multiple valuations" track at LAEMOS in Chile 2016 and the $\mathrm{PhD}$ valuation group at CBS for useful and inspiring comments on previous versions of the paper.

\section{References}

Bakhtin, Mikhail M. 1937. "Forms of Time and of the Chronotope in the Novel: Notes Toward a Historical Poetics." In Narrative Dynamics: Essays on Time, Plot, Closure, and Frames, edited by Brian Richardson, 15-24. Ohio: The Ohio State University Press.

Barley, Stephen R. 1986. "Technology as an Occasion for Structuring: Evidence from Observations of CT Scanners and the Social Order of Radiology Departments." Administrative Science Quarterly 31(1): 78108.

Barnard, Chester I. 1968 [1938]. The Functions of the Executive. Cambridge, MA and London: Harvard University Press.

Bech, Karsten. 2012. "Landsdækkende Undersøgelse Af Patientoplevelserrespekter Patienterne.” Ugeskr Loger 174: 40.

Boltanski, Luc, and Laurent Thévenot. 1999. "The Sociology of Critical Capacity." European Journal of Social Theory 2(3): 359-377.

Boltanski, Luc, and Laurent Thévenot. 2006. On Justification: Economies of Worth. Princeton, NJ: Princeton University Press.

Brandao de Souza, Luciano. 2009. "Trends and Approaches in Lean Healthcare." Leadership in Health Services 22(2): 121.

Cabantous, Laure, and Théodora Dupont-Courtade. 2015. "What is a Catastrophe Model Worth?" in Making Things Valuable, edited by Martin Kornberger, Lise Justesen, Anders Koed Madsen and Jan Mouritsen, 167-186. Oxford: Oxford University Press.

Callon, Michel. 2007. "What does it Mean to Say that Economics is Performative?" in Do Economists make Markets, edited by Donald MacKenzie, Fabian Muniesa and Lucia Siu, 311-357. Princeton and Oxford: Princeton University Press.

Cochoy, Franck. 2008. "Calculation, Qualculation, Calqulation: Shopping Cart Arithmetic, Equipped Cognition and the Clustered Consumer." Marketing Theory 8(1): 15-44.

Deleuze, Gilles. 1993. The Fold: Leibniz and the Baroque. Minneapolis, MN: University of Minnesota Press.

Doganova, Liliana, Martin Giraudeau, Claes-Fredrik Helgesson, Hans Kjellberg, Francis Lee, Alexandre Mallard, Andrea Mennicken, Fabian Muniesa, Ebba Sjögren, and Teun Zuiderent-Jerak. 2014. "Valuation Studies and the Critique of Valuation." Valuation Studies 2(2): 87-96. 
Dussauge, Isabelle, Claes-Fredrik Helgesson, and Francis Lee. 2015. Value Practices in the Life Sciences and Medicine. Oxford: Oxford University Press.

Edwards, Kasper, and Anders Paarup Nielsen. 2011. "Improving Healthcare through Lean Management: Experiences from the Danish Healthcare System." NOHR2011 - 5th Nordic Conference on Health Organization and Management, Frederiksberg, January.

Espeland, Wendy Nelson, and Michael Sauder. 2007. "Rankings and Reactivity: How Public Measures Recreate Social Worlds1." American Journal of Sociology 113(1): 1-40.

Foucault, Michel. 1988. Politics, Philosophy, Culture, Ed. LD Kritzman. New York and London: Routledge.

Fourcade, Marion. 2011. "Cents and Sensibility: Economic Valuation and the Nature of 'Nature'." American Journal of Sociology 116(6): 1721-1777.

Heuts, Frank, and Annemarie Mol. 2013. "What is a Good Tomato? A Case of Valuing in Practice." Valuation Studies 1(2): 125-146.

Holm, Petter, and Kåre Nolde Nielsen. 2007. "Framing Fish, Making Markets: The Construction of Individual Transferable Quotas (ITQs)." The Sociological Review 55(s2): 173-195.

Imai, Masaaki. 1986. Kaizen. Vol. 201. New York: Random House Business Division.

Kornberger, Martin, Lise Justesen, Anders Koed Madsen, and Jan Mouritsen. 2015. Making Things Valuable. Oxford: Oxford University Press.

Krafve, Linus Johansson. 2015. Valuation in Welfare Markets: The Rule Books, Whiteboards and Swivel Chairs of Care Choice Reform. Dissertation. Linköping: Linköping University Electronic Press.

Lamont, Michèle. 2012. "Toward a Comparative Sociology of Valuation and Evaluation.” Sociology 38(1): 201.

Latour, Bruno, and Michel Callon. 1997. “Thou Shall Not Calculate!'or How to Symmetricalize Gift and Capital.” Trans. Javier Krauel, French Title: Tu ne calculeras pas!" ou comment symétriser le don et le capital. Revue du MAUSS 9: 45-70.

Latour, Bruno, and Vincent Antonin Lépinay. 2009. The Science of Passionate Interests: An Introduction to Gabriel Tarde's Economic Anthropology. Chicago, IL: Prickly Paradigm Press.

Latour, Bruno, and Couze Venn. 2002. "Morality and Technology the End of the Means." Theory, Culture \& Society 19(5-6): 247-260.

Lawrence, Paul R., and Jay W. Lorsch. 1967. "Differentiation and Integration in Complex Organizations." Administrative Science Quarterly 12(1): 147.

Lévi-Strauss, Claude. 1963. Structural Anthropology. Vol. 1. New York: Basic Books.

Lyotard, Jean-François. 1993. Political Writings. Minneapolis, MN: University of Minnesota Press.

Madsen, Marie Henriette. 2015. Emerging and Temporary Connections in Quality Work. Frederiksberg: Copenhagen Business School. 


\section{Valuation Studies}

Pamela Mazzocato, Terese Stenfors-Hayes, Ulrica von Thiele Schwarz, Henna Hasson, Monica Elisabeth Nyström 2016. "Kaizen Practice in Healthcare: A Qualitative Analysis of Hospital Employees' Suggestions for Improvement." BMJ Open 6(7): 1-8.

Mintzberg, Henry. 1979. The Structuring of Organizations - A Synthesis of the Research. Englewood Cliffs, NJ: Prentice-Hall.

Orlikowski, Wanda J., and Susan V. Scott. 2013. "What Happens when Evaluation Goes Online? Exploring Apparatuses of Valuation in the Travel Sector." Organization Science 25(3): 868-891.

Owen, David. 1995. "Genealogy as Examplary Critique: Reflections on Foucault and the Imagination of the Political." International Journal of Human Resource Management 24(4): 489-506.

Pénet, Pierre. 2015. "Rating Reports as Figuring Documents. How Credit Rating Agencies Build Scenarios of the Future". In Making Things Valuable, edited by Martin Kornberger, Lise Justesen, Anders Koed Madsen and Jan Mouritsen, 62-88. Oxford: Oxford University Press.

Perrow, Charles. 1970. Organizational Analysis: A Sociological View. Belmont, CA: Wadsworth.

Radnor, Zoe. 2011. "Debate: How Mean is Lean really?” Public Money \& Management 31(2): 89-90.

Radnor, Zoe J., Matthias Holweg, and Justin Waring. 2012. "Lean in Healthcare: The Unfilled Promise?" Social Science \& Medicine 74(3): 364-371.

Rona-Tas, Akos, and Stefanie Hiss. 2011. "Forecasting as Valuation.” In The Worth of Goods: Valuation and Pricing in the Economy, edited by Jens Beckert and Patrik Aspers, 223-246. Oxford: Oxford University Press.

Running, Alice. 1997. "Snapshots of Experience: Vignettes from a Nursing Home." Journal of Advanced Nursing 25(1): 117-122.

Schein, Edgar H. 1965. Organizational Psychology. Englewood Cliffs, NJ: Prentice-Hall.

Scott, W. Richard. 1981. "Developments in Organization Theory, 1960-1980.” American Behavioral Scientist 24(3): 407-422.

Simon, Herbert A. 1957. Models of Man; Social and Rational. Oxford: Wiley.

Simon, Herbert A. 1964. "On the Concept of Organizational Goal." Administrative Science Quarterly 9(1): 1-22.

Sjögren, Ebba. 2008. "Deciding Subsidy for Pharmaceuticals Based on Ambiguous Evidence." Journal of Health Organization and Management 22(4): 368-383.

Stark, David. 2011. "What's Valuable?" In The Worth of Goods - Valuation and Pricing in the Economy, edited by Jens Beckert and Patrik Aspers, 319-338. Oxford: Oxford University Press:.

Thévenot, Laurent. 2007. "The Plurality of Cognitive Formats and Engagements Moving between the Familiar and the Public." European Journal of Social Theory 10(3): 409-423. 
Thévenot, Laurent. 2015. "Making Commonality in the Plural, on the Basis of Binding Engagements." In Social Bonds as Freedom: Revising the Dichotomy of the Universal and the Particular, 82-108. New York: Berghahn.

Thompson, James D. 2007 [1967]. Organizations in Action: Social Science Bases of Administrative Theory. Classics in Organization and Management Series, 5th ed. New Brunswick, NJ: Transaction.

Thompson, James D. 2001 [1967]. "Organizations in Action.” In Classics of Organization Theory, edited by Jay M. Shafritz, J. Steven Ott, and Yong Suk Jang, 268-281. Boston, MA: Cengage Learning.

Timmermans, Stefan, and Marc Berg. 2003. The Gold Standard: The Challenge of Evidence-Based Medicine and Standardization in Health Care. Philadelphia, PA: Temple University Press.

van Loon, Esther, and Roland Bal. 2014. "Uncertainty and the Development of Evidence-Based Guidelines." Valuation Studies 2(1): 43-64.

Vikkelsø, Signe. 2010. "Mobilizing Information Infrastructure, Shaping Patient-Centred Care." International Journal of Public Sector Management 23(4): 340-352.

Zuiderent-Jerak, Teun, and Marc Berg. 2010. "The Sociology of Quality and Safety in Health Care: Studying a Movement and Moving Sociology.” In The Handbook of Medical Sociology, edited by Chloe E. Bird, P Conrad, Allen M. Fremont and Stefan Timmermans, 324-337. Nashville, TN: Vanderbilt University Press.

Zuiderent-Jerak, Teun, and Stans van Egmond. 2015. "Ineffable Cultures or Material Devices: What Valuation Studies can Learn from the Disappearance of Ensured Solidarity in a Health Care Market." Valuation Studies 3(1): 45-73.

Zuiderent-Jerak, Teun, Kor Grit, and Tom van der Grinten. 2015. “Critical Composition of Public Values: On the Enactment and Disarticulation of what Counts in Health-Care Markets." In Value Practices in the Life Sciences and Medicine, edited by Claes-Frederik Helgesson, Francis Lee and Isabelle Dussauge, 119-135. Oxford: Oxford University Press.

Amalie Martinus Hauge is interested in the interplay between values, management technologies and organizations in the public sector. Her $\mathrm{PhD}$ is an ethnographic study of what happens to values and value practices when a children's hospital in Denmark introduces Lean management. 